\title{
Computer Simulation of PAN/PVP Blends Compatibility and Preparation of Aligned PAN Porous Nanofibers via Magnetic-Field-Assisted Electrospinning PAN/PVP Blends
}

\author{
Linyu MEI ${ }^{1 *}$, Xigang WANG ${ }^{2}$, Yaqing LIU $^{3}$, Junyuan WANG ${ }^{1}$ \\ ${ }^{1}$ School of Mechanical Engineering, North University of China, Taiyuan 030051, China \\ ${ }^{2}$ Department of Mechanical Engineering, Taiyuan Institute of Technology, Taiyuan 030051, China \\ ${ }^{3}$ Shanxi province Key laboratory of Functional Nanocomposites, School of Materials Science and Engineering, North \\ University of China, Taiyuan 030051, China \\ crossref http://dx.doi.org/10.5755/j01.ms.25.1.18794
}

Received 09 August 2017; accepted 31 January 2018

\begin{abstract}
Binary blend compatibility of polyacrylonitrile (PAN) and polyvinylpyrrolidone (PVP) was computationally simulated at both molecular and mesoscopic levels in order to provide theoretical support for preparing PAN porous nanofibers from PAN/PVP blends. In molecular simulation, Flory-Huggins interaction parameters were calculated to estimate the blend compatibility, in which PAN and PVP were found to be immiscible. This had been further validated by the mesoscopic simulation in terms of the free energy density, the order parameters, and the mesoscopic morphology. Aligned PAN porous nanofibers were prepared by selectively removing PVP from the PAN/PVP blend nanofibers which was prepared by Magnetic-field-assisted electrospinning (MFAES).

Keywords: computer simulation, compatibility, electrospinning, aligned porous nanofibers, PAN.
\end{abstract}

\section{INTRODUCTION}

Porous electrospun fibers have great potential for practical applications in many fields such as energy [1-4], environment [5] and healthcare [6]. The remarkable advantage of porous nanofibers is the higher specific surface area, which makes them ideal candidates for filtration media $[7,8]$, absorbents [9], and catalyst supports [10, 11]. In the field of production of scaffolds for tissue engineering, porous surfaces have been demonstrated to favor cell attachment, cell proliferation and cell spreading on the scaffold $[12,13]$.

There are two ways to prepare porous fibers [14]. One is through controlling electrospinning condition such as strong volatile solvent and a certain relative humidity or proper post-treatments. Another is achieved by electrospinning two immiscible components in a common solvent followed by selectively removing the phaseseparated component. Polyacrylonitrile (PAN) is an important polymer and widely used for filtration, adsorption and composite materials. Because of the strong intermolecular interaction of the chains, PAN can only dissolve in strong polar solvents. It is not easy to obtain porous structures simply by controlling electrospinning parameters for PAN, which can only dissolve in common polar solvents with high boiling points. In this study, we prepared PAN porous nanofibers by selectively removing polyvinylpyrrolidone (PVP) from PAN/PVP blend nanofibers.

This selective removal method is based on the premise that the blend of two polymers is immiscible. Various methods including viscosity measurement, differential scanning calorimetry (DSC), and Fourier transform infrared (FTIR) spectroscopy have been previously used to explore the compatibility between two polymers $[15,16]$. The experimental protocols are time-consuming and expensive. Unfortunately, contradictory results can be found in the literature for certain systems [16]. With the ever-growing computational power and resource, molecular simulations have played an important role in materials modeling and subsequent technology development, which can provide valuable microscopic and mesoscopic insights into the phase morphology and the interfacial behaviors of the immiscible molecules $[17,18]$. In this study, molecular dynamics (MD) and mesoscopic dynamics (MesoDyn) simulations have been performed on PAN/PVP polymer blends to investigate their miscibility/immiscibility and mesoscopic morphologies

In general, well-aligned, one-dimensional nanostructures exhibit much more excellent properties compared to their disordered counterparts [19]. The aligned nanofibers have great promising application potential in the information technology field such as field-effect transistors (FETs) [20], light-emitting diodes (LEDs) [21], solar cells (SCs) [22], highly sensitive sensors [23], logic computations [24], microcircuits [25] as well as in other application fields $[12,13]$. It has been aware of that the aligned fibers may play an important role in tissue engineering, such as for repairing tendon and ligaments, blood vessel, and neural tissues, due to the similarity between the aligned topography of fibers in the natural extracellular matrix of these tissues and that of electrospun scaffolds [26, 27]. Researchers have demonstrated that stem cells grow along the long axes of aligned electrospun fibers.

\footnotetext{
${ }^{*}$ Corresponding author. Tel.: +86-351-3922102; fax: +86-351-3922102.

E-mail address: mly81@163.com (L. Mei)
} 
Meanwhile, the existence of pores can increase surface area of nanofibers and promote cell adhesion as previously reported [28]. PAN has been prepared into aligned nanofibers [29] and porous nanofibers [30]. However, aligned PAN nanofibers with porous structures have not been reported yet.

The aim of this work was to investigate the binary blend compatibility of PAN and PVP using MD and MesoDyn simulation methods in order to provide theoretical support for experimental fabrication of the aligned PAN porous nanofibers. Sequentially, the aligned PAN porous nanofibers were successfully prepared by selectively removing PVP from the blend nanofibers using magneticfield-assisted electrospinning (MFAES) methods.

\section{SIMULATION AND EXPERIMENTAL}

\subsection{Computer simulation strategies of the compatibility of PAN and PVP}

Simulations of PAN and PVP were performed using Materials Studio 7.0. Twenty-seven repeating units for PAN and Twenty-six for PVP were selected to create the initial amorphous cell of polymer blend. The blend system was initially subjected to energy minimization. The MD simulation was then performed under the COMPASS force field for $1000 \mathrm{ps}$ in the NPT ensemble and $500 \mathrm{ps}$ in the NVT ensemble. The time step was 1 fs. The last 200 ps trajectories were used for data analysis. During the whole simulation process, the temperature and pressure were maintained using the Berendsen method. The snapshot of PAN/PVP blends with 80/20, 60/40, 50/50, 40/60 compositions are shown in Fig. 1.

In order to illustrate the phase separation dynamics at the mesoscopic level, MesoDyn program was used. The principle and method of MesoDyn simulation were reported in our previous work [18]. The characteristic ratios of PAN and PVP were 7.326 and 9.900, respectively. The MesoDyn input parameters were taken from the above MD simulation results of PAN/PVP blends at different compositions. In accordance with experiment data, the degrees of polymerization of PAN and PVP have been chosen at 1698 and 270 and therefore, $\mathrm{N}_{\text {meso }}$ [18] taken for PAN and PVP were 231 and 27, respectively. The bond length was $1.1543 \mathrm{~nm}$, and the time step was $50 \mathrm{~ns}$. In process of the entire simulation, the noise parameter was maintained at 75.002 to guarantee the system stability. The grid dimensions were chosen to be $32 \times 32 \times 32 \mathrm{~nm}$ and the size

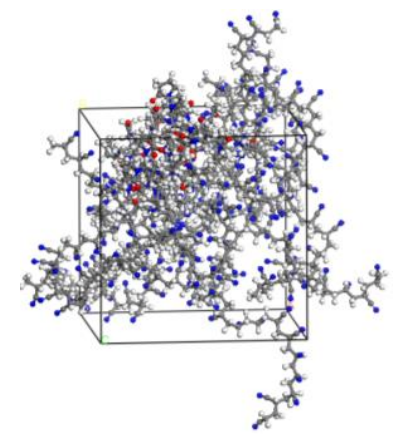

a

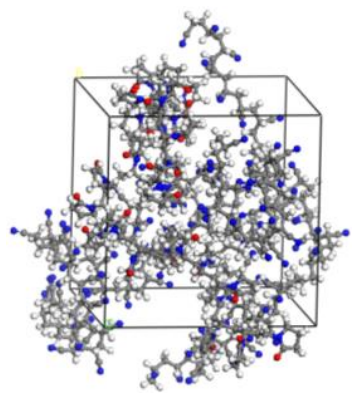

b of the mesh over which density variations are to be plotted in MesoDyn length units using the Grid spacing field of $1 \mathrm{~nm}$.

\subsection{Experimental preparation of aligned PAN porous nanofibiers}

\subsubsection{Materials}

PAN powder with a molecular mass of 90000 and PVP powder with a molecular mass of 1000000 were supplied by Spectrum China Co., Ltd, China and Shanghai WellTone Material Technology Co. The solvent used for dissolving PAN/PVP was N,N-dimethylformamide (DMF, Tianjin Fengchuan Chemical Reagent Science And Technology Co.). PAN/PVP/DMF solution of different mixing ratios was prepared in a glass reactor, using an overhead mixer with impeller. During stirring, the reactor was placed in a $40{ }^{\circ} \mathrm{C}$ water bath for $24 \mathrm{~h}$.

\subsubsection{MFAES of PAN/PVP fibers}

Fig. 2 shows a schematic illustration of the setup used in MFAES process. Two bar magnets $(7.7 \mathrm{~cm} \times 1.7 \mathrm{~cm} \times 5.7 \mathrm{~cm})$ were introduced to the conventional collecting region. The PAN/PVP solution was contained in a plastic syringe $(20 \mathrm{~mL})$ fitted with a plane tip of steel needle with an inner diameter of $700 \mu \mathrm{m}$. The needle was connected to a high-voltage power supply (DW-P503IACDF, Tianjin Dongwen High-Voltage Power Supply Co., Ltd). The feeding rate of the PAN solution into the tip was controlled at $1 \mathrm{~mL} / \mathrm{h}$ using a syringe pump (KDS LEGATO 200, microplus technology (Shanghai instrument co., LTD). The distance between the tip of the needle and the collector was $12 \mathrm{~cm}$. The MFAES of PAN was performed at $25 \pm 2{ }^{\circ} \mathrm{C}$ and relative humidity of $25-30 \%$.

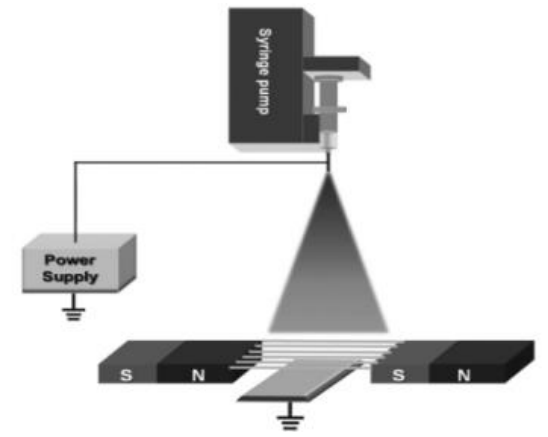

Fig. 2. Schematic illustration of the setup used in the MFAES method [31]
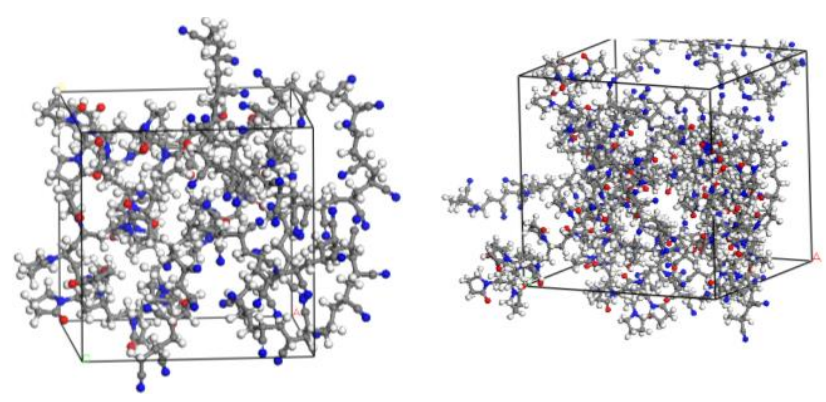

c

Fig. 1. Snapshot of amorphous cells for PAN/PVP blends of different compositions: $a-80 / 20 ; b-60 / 40 ; c-50 / 50 ; d-40 / 60$ 


\subsubsection{Preparation of aligned PAN porous fibers}

The prepared hybrid fiber membranes were stuck on slides carefully and dried in a vacuum oven at $60{ }^{\circ} \mathrm{C}$ for $24 \mathrm{~h}$. The resulting fibrous membranes were placed in $90{ }^{\circ} \mathrm{C}$ deionized water for at least $10 \mathrm{~h}$ to remove the PVP. The treated fibrous membranes were then dried in a vacuum at $40{ }^{\circ} \mathrm{C}$ for $24 \mathrm{~h}$ for further analysis.

\subsubsection{Morphology observation}

Scanning electron microscopy (SEM, Tescan MIRA 3LMH, Czech) was used to characterize the morphologies of the fibers.

\section{RESULTS AND DISCUSSION}

\subsection{Flory-Huggins interaction parameter}

The Flory-Huggins interaction parameter $\left(\chi_{\mathrm{AB}}\right)$ was used to estimate the compatibility/incompatibility, which could be given by $[18,32]$ :

$$
\chi_{A B}=\left(\frac{\Delta E_{m i x}}{R T}\right) V_{m},
$$

where $V_{m}$ is the molar volume of the repeat. $\Delta E_{m i x}$ is the energy of mixing calculated as $[18,32]$ :

$$
\Delta E_{\text {mix }}=\phi_{A}\left\{\frac{E_{c o h}}{V}\right\}_{A}+\phi_{B}\left\{\frac{E_{c o h}}{V}\right\}_{B}-\left\{\frac{E_{c o h}}{V}\right\}_{\text {mix }} \text {. }
$$

In the above equations, subscripts $A, B$ and mix represent the $C E D$ values of PAN, PVP and their blends by considering the identity $C E D=E_{c o h} / V$. Symbols $\varphi_{\mathrm{A}}$ and $\varphi_{\mathrm{B}}$ represent the volume fractions of PAN and PVP. With these two equations, the curve of Flory-Huggins interaction parameter versus weight of PVP was finally obtained (Fig. 3).

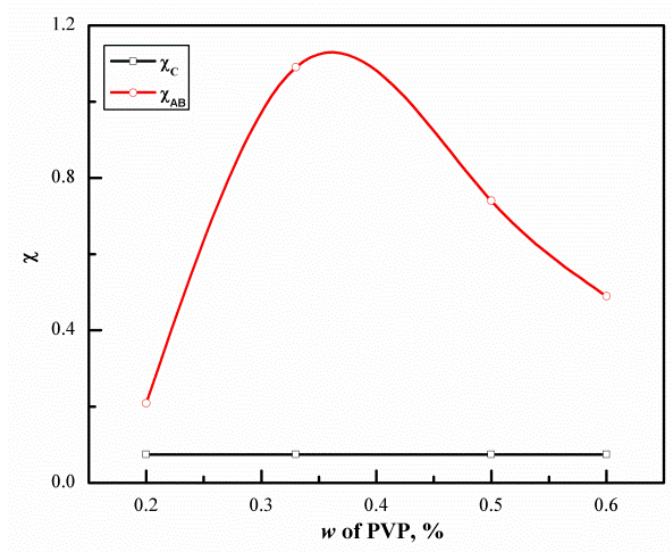

Fig. 3. Flory-Huggins interaction parameter $\left(\chi_{A B}\right) v s$. mass fraction of PVP

A positive value of the Flory-Huggins interaction parameter $\chi_{\mathrm{AB}}$ indicates immiscibility for blends of high molecular weight polymers, but in general, the critical value of $\chi_{C}$ obeys by [33]:

$\chi_{c}=\frac{1}{2}\left(\frac{1}{\sqrt{n_{A}}}+\frac{1}{\sqrt{n_{B}}}\right)^{2}$, where $n_{A}$ and $n_{B}$ represent the degree of polymerization of the pure polymers. If $\chi_{A B}$ of the blend is smaller than $\chi_{c}$, the system is miscible, then the conclusion of compatibility could be safely obtained. If $\chi_{A B}$ is greater than the critical value, the blend exhibits partial miscibility. If $\chi_{A B}$ is considerably larger than the critical one, then the blends are incompatible which means they would form two separate phases. In this case, for all proportion compositions of PAN/PVP blends, the $\chi_{A B}$ are all above the critical value, indicating immiscibility of PAN and PVP blends.

\subsection{Free energy density and order parameter}

The MesoDyn program was also used to simulate the phase separation dynamics of the blends at the mesoscipic level. In MesoDyn simulations, it is important to realize the system stability before one gets accurate and reliable data. The free energy density should asymptotically approach a stable value after reaching dynamic equilibrium. The evolution of free energy could be used to evaluate the stability of a blend system. However, the free energy density is not routinely calculated for real systems. Hence, the direct comparison with experimental data is not possible [33]. Plots of free energy density versus time step given in Fig. 4 show that the system is stable after equilibrium being reached at later stages.

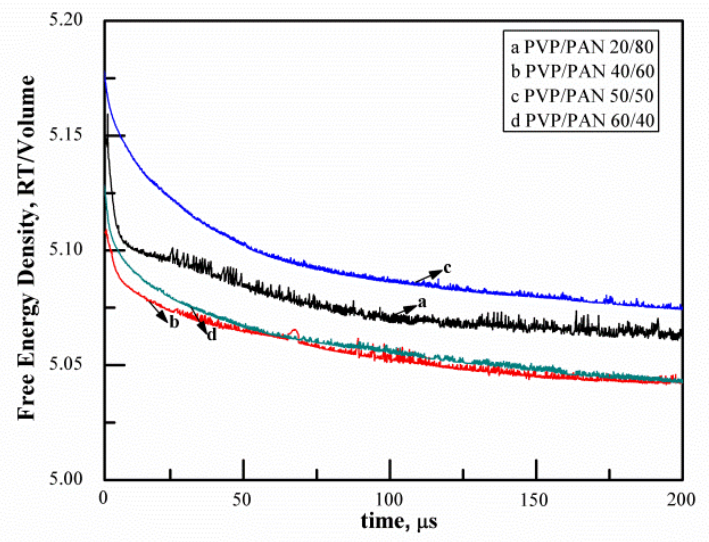

Fig. 4. The evolution of free energy density $v$ s. time steps for PAN/PVP blends

The order parameter $\left(\mathrm{P}_{\mathrm{i}}\right)$ was defined as the average volume of the difference between local density squared and the overall density squared, which is an important parameter to understand the miscibility/immiscibility aspects of polymer blends. Fig. 5 shows the plot of order parameters of PVP for different PAN/PVP blends. Generally, if the order parameter exceeds 0.1 , the polymer blends can be considered to be immiscible. With the order parameter being calculated and compared to 0.1 , the miscibility was proved. The order parameters with large values indicated strong phase segregation. Conversely, very small values indicated good miscibility of blends. For all proportion compositions of PAN/PVP blends considered, the order parameters were $>0.1$, indicating immiscibility of blends.

\subsection{Mesoscopic morphology of PAN/PVP blends}

Fig. 6 illustrates the morphologies of PAN/PVP blends. The green color corresponds to areas of pure PVP, while the red color areas correspond to the other component PAN. 
Different levels of phase separation phenomena were observed in PAN/PVP blends which were caused by repulsion between different polymer beads.

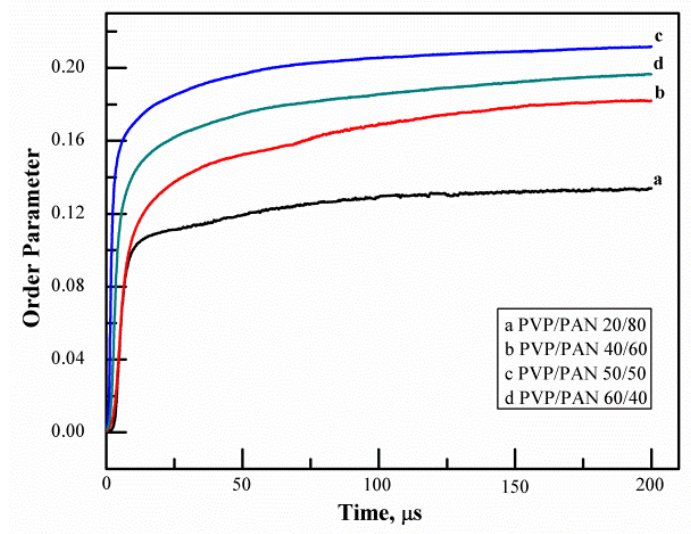

Fig. 5. The order parameters of PVP for PAN/PVP blends $v s$. time steps

It is distinct that the morphology consisting of PVP globules dispersed in PAN matrix for blends with predominant PAN content (i.e. 80/20). With increasing PVP composition, a deformation of the dispersed phase appears. PVP displays a thread-like morphology with some globules dispersed in the PAN matrix. The PAN-PVP specimen at $50 / 50$ and 40/60 blend ratios show a co-continuous twophase interpenetrating morphology. It is also notable that in the cases of 80/20, 60/40, 50/50, 40/60 PAN/PVP blends, the domain surfaces of dispersed phase appear to be very smooth, suggesting a poor interfacial adhesion in the blends. This also validates the results obtained from atomistic simulations discussed before.

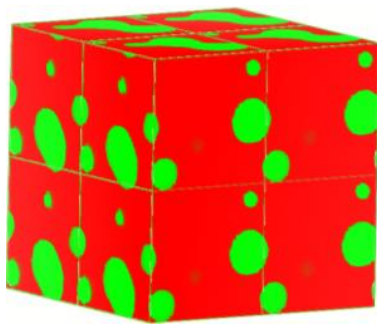

a

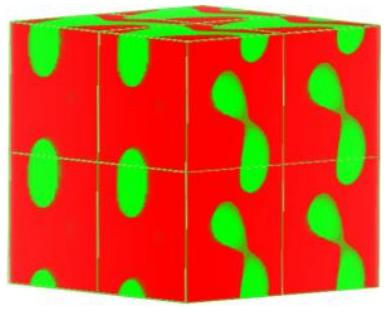

b

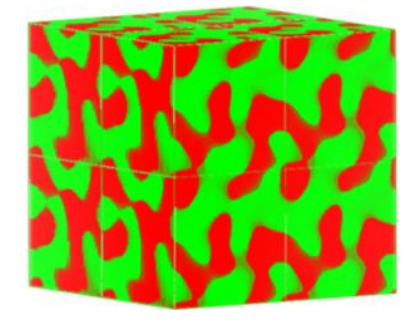

c
From the foregoing result, it can be seen that PAN/PVP blends are immiscible at different mixture ratios. Hence, we can prepare PAN porous nanofibers by selectively removing PVP from the PAN/PVP blend nanofibers.

\subsection{MFAES aligned PAN/PVP fibers}

The concentration of the solution as well as the applied voltage and distance between the charged electrode and the grounded target were adjusted in order to obtain aligned hybrid nanofibers. Fig. 7 displays SEM photographs of the electrospun composite nanofiber fabricated at different blend ratios.

We can see from Fig. 7 that all the PAN/PVP/DMF solutions at different blend ratios have good spinnability and they form smooth, free of beads, and uniform nanofibers. Meanwhile, it demonstrates that highly ordered hybrid nanofibers are able to be prepared by MFAES.

\subsection{Aligned PAN porous fibers}

After washing, all of the nanofiber samples with long and continuous cylindrical morphologies arrange regularly/perfectly as shown in Fig. 8. It clearly shows that abundant nanopores of narrow elliptical shape are formed on the fiber surface. The surface porous structure can maintain the excellent mechanical properties of nanofibers. The density and size of nanopores, can be tuned by changing the spinning solution proportioning, increasing with the increase of PVP content. In addition to the morphology change of the fiber surface, the diameter of the electrospun fibers also decrease. In particular, the nanofibers still hold well orderly structure after washing. The present study clearly shows that the method shown here is not only simple but also effective to create the aligned porous nanofibers.

Fig. 6. Isosurface of density fields for PAN/PVP blends of different compositions: $a-80 / 20 ; b-60 / 40 ; c-50 / 50 ; d-40 / 60$
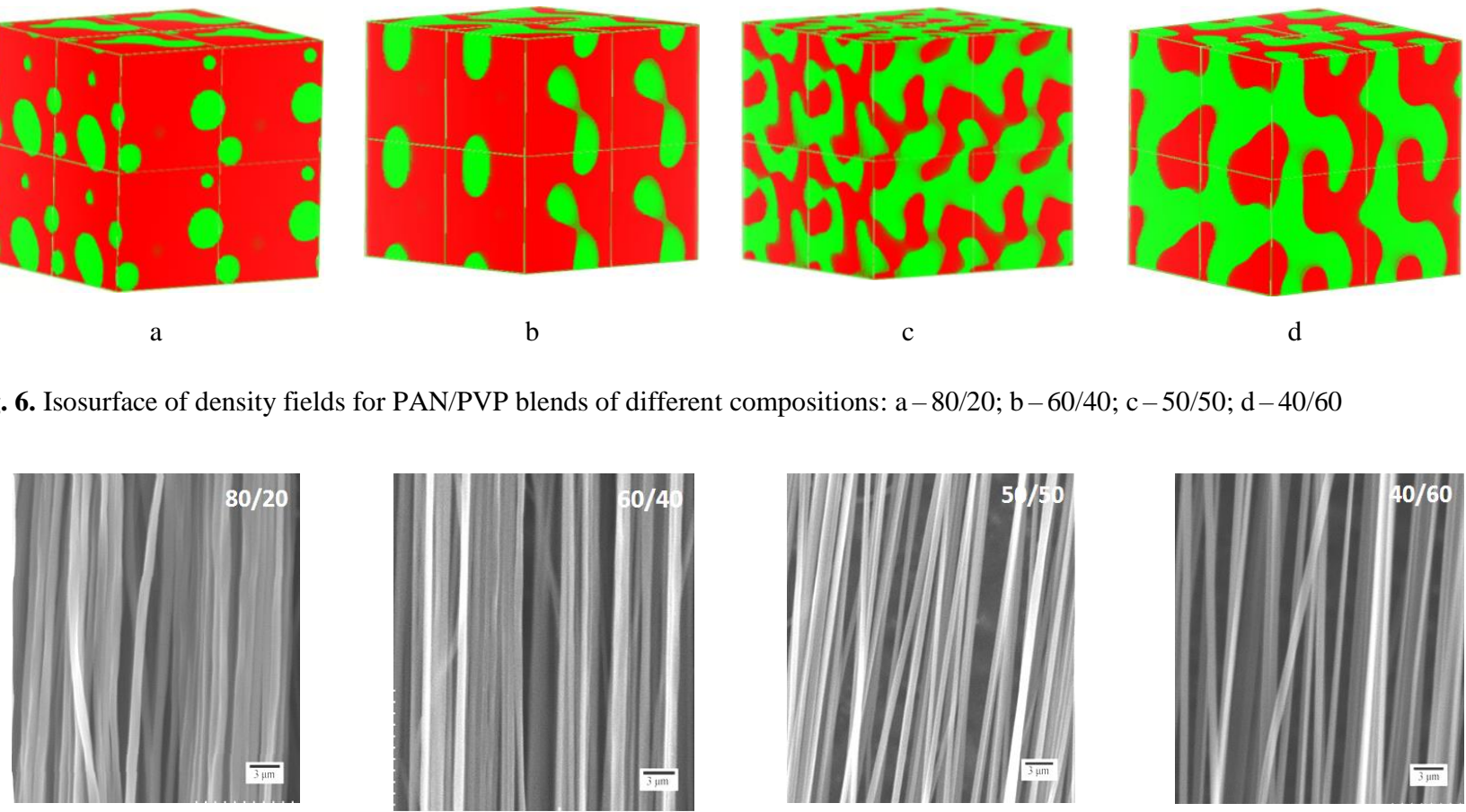

d

Fig. 7. SEM image of PAN/PVP nanofibers under blend ratios of 80/20, 60/40, 50/50, 40/60 

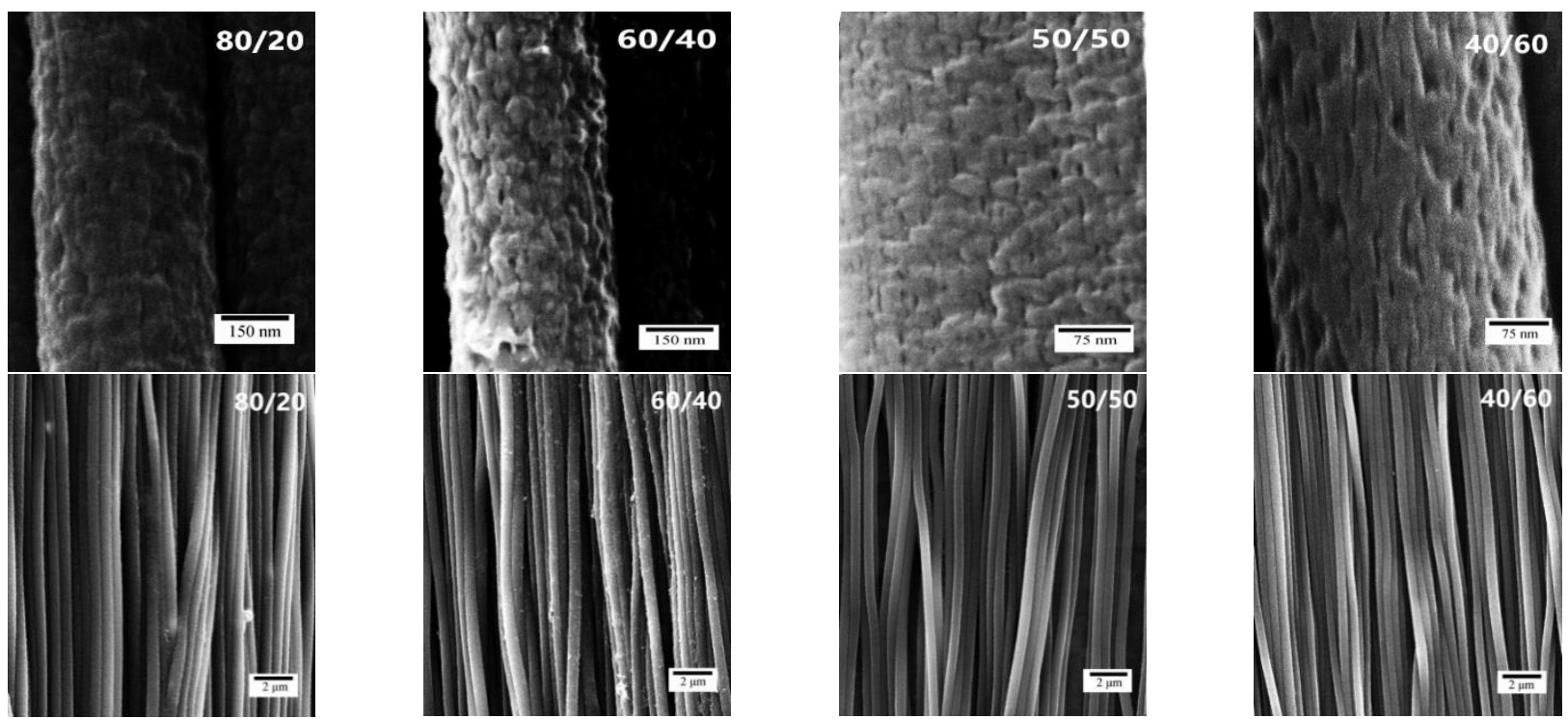

Fig. 8. SEM image of aligned PAN porous fibers prepared from different proportions PAN/PVP fibers of 80/20, 60/40, 50/50 and 40/60

\section{CONCLUSIONS}

The compatibility study of PAN/PVP by molecular dynamics and mesoscopic dynamics simulations shows the immiscibility of blends which provides theoretical support for a physical preparation of aligned PAN porous fibers obtained from PAN/PVP compound fibers. This study demonstrates the efficacy of combining the virtual simulation with experimental realization in the production of PAN fibers. Our method provides an effective way to prepare aligned porous nanofibers by MFAES, suitable for those polymers which can only dissolve in polar solvents with high boiling points. The PAN porous fibers with highly ordered structure as prepared in this paper may have substantial application potential which will require a further investigation in the future study.

\section{Acknowledgments}

The authors thank Prof. Feng Liu and Dr. Congyun Zhang for their helpful suggestions and useful comments on the manuscirpt. Also, they gratefully acknowledge the financial support from the natural science foundation for Young Scientists of ShanXi Province, China (Grant No. 2015021068).

\section{REFERENCES}

1. Zhou, Y.Q., Shen, Y., Li, Y.H., Zuo, Q.H., Duan, Q. Fabrication, Formation Mechanism and the Application in Lithium-Ion Battery of Porous $\mathrm{Fe}_{2} \mathrm{O}_{3}$ Nanotubes via SingleSpinneret Electrospinning Electrochimica Acta 158 2015: pp. $105-112$. https://doi.org/10.1016/j.electacta.2015.01.149

2. Miao, Y.E., Huang, Y.P., Zhang, L.S., Fan, W., Lai, F.L., Liu, T.X. Electrospun Porous Carbon Nanofiber@MoS2 Core/Sheath Fiber Membranes as Highly Flexible and BinderFree Anodes for Lithium-Ion Batteries Nanoscale 7 (25) 2015: pp. $11093-11101$. https://doi.org/10.1039/C5NR02711J

3. Li, W.H., Li, M.S., Wang, M., Zeng, L.C., Yu, Y. Electrospinning with Partially Carbonization in Air: Highly Porous Carbon Nanofibers Optimized for High-Performance
Flexible Lithium-Ion Batteries Nano Energy 13 2015: pp. $693-701$.

https://doi.org/10.1016/j.nanoen.2015.03.027

4. Kim, B.H., Yang, K.S. Enhanced Electrical Capacitance of Tetraethyl Orthosilicate-Derived Porous Carbon Nanofibers Produced via Electrospinning Journal of Electroanalytical Chemistry 714 2014: pp. 92-96.

https://doi.org/10.1016/j.jelechem.2013.12.019

5. Guo, Z.Y., Huang, J.T., Xue, Z.H., Wang, X.M. Electrospun Graphene Oxide/Carbon Composite Nanofibers with Well-Developed Mesoporous Structure and Their Adsorption Performance for Benzene and Butanone Chemical Engineering Journal 306 2016: pp. 99-106. https://doi.org/10.1016/j.cej.2016.07.048

6. Entekhabi, E., Nazarpak, M.H., Mortatzadeh, F., Sadeghi, A. Design and Manufacture of Neural Tissue Engineering Scaffolds Using Hyaluronic Acid and Polycaprolactone Nanofibers with Controlled Porosity Materials Science \& Engineering C-Materials for Biological Applications 69 2016: pp. 380-387. https://doi.org/10.1016/j.msec.2016.06.078

7. Song, B.T., Xu, C. Highly Hydrophobic and Superoleophilic Nanofibrous Mats with Controllable Pore Sizes for Efficient Oil/Water Separation Langmuir 32 2016: pp. 9960-9966. https://doi.org/10.1021/acs.langmuir.6b02500

8. Zhang, S.C., Liu, H., Xia, Y., Yu, J.Y. Anti-deformed Polyacrylonitrile/Polysulfone Composite Membrane with Binary Structures for Effective Air Filtration ACS Applied Materials \& Interfaces 8 2016: pp. 8086-8095. https://doi.org/10.1021/acsami.6b00359

9. Zhang, Y., Shi, R.X., Yang, P., Song, X.L., Zhu, Y.N., Ma, Q. Fabrication of Electronspun Porous $\mathrm{CeO} 2$ Nanofibers with Large Surface Area for Pollutants Removal Ceramics International 42 2016: pp. 14028-14035. https://doi.org/10.1016/j.ceramint.2016.06.009

10. Berenguer, R., Fornells, J., Garcia-Mateos, F.J., Guerrero-Perez, M.O., Rodriguez-Mirasol, J., Cordero, T. Novel Synthesis Method of Porous VPO Catalysts with Fibrous Structure by Electrospinning Catalysis Today 277 2016: pp. 266-273. https://doi.org/10.1016/j.cattod.2016.03.002 
11. Ma, X.H., Gu, S.W., Xu, Z.L. Structure and Property of PFSA/PES Porous Catalytic Nanofibers Catalysis Today 276 2016: pp. $133-138$. https://doi.org/10.1016/j.cattod.2016.01.024

12. Sperling, L.E., Reis, K.P., Pranke, P., Wendorff, J.H. Advantages and Challenges Offered by Biofunctional CoreShell Fiber Systems for Tissue Engineering and Drug Delivery Drug Discovery Today 21 2016: pp. 1243-1256. https://doi.org/10.1016/j.drudis.2016.04.024

13. Ngadiman, N.H.A., Yusof, N.M., Idris, A., Misran, E., Kumiawan, D. Development of Highly Porous Biodegradable Gamma-Fe2O3/Polyvinyl Alcohol Nanofiber Mats Using Electrospinning Process for Biomedical Application Materials Science \& Engineering C-Materials for Biological Applications 70 2017: pp. 520-534. https://doi.org/10.1016/j.msec.2016.09.002

14. Yu, X.L., Xiang, H.F., Long, Y.H., Zhao, N., Zhang, X.L., Xu, J. Preparation of Porous Polyacrylonitrile Fibers by Electrospinning a Ternary System of PAN/DMF/H2O Materials Letters 64 2010: pp. 2407-2409. https://doi.org/10.1016/j.matlet.2010.08.006

15. Rakkapao, N., Vao-soongnern, V. Molecular Simulation and Experimental Studies of the Miscibility of Chitosan/Poly(Ethylene Oxide) Blends Journal of Polymer Research 21 2014: pp. 606-610. http://dx.doi.org/10.1007/s10965-015-0880-6

16. Gu, C.H., Gu, H.Y., Lang, M.D. Molecular Simulation to Predict Miscibility and Phase Separation Behavior of Chitosan/Poly(Epsilon-Caprolactone) Binary Blends: A Comparison with Experiments Macromolecular Theory and Simulations 22 2013: pp. 377-384. https://doi.org/10.1002/mats.201300109

17. Nawaz, M., Baloch, M.K., Rehman, W. Investigating the Compatibility of Polymers in Common Solvent Journal of the Chilean Chemical Society 55 2010: pp. 90-93. http://dx.doi.org/10.4067/S0717-97072010000100021

18. Fu, Y.Z., Liao, L.Q., Lan, Y.H., Yang, L.X., Mei, L.Y., Liu, Y.Q., Hu, S.Q. MD Molecular Dynamics and Mesoscopic Dynamics Simulations for Prediction of Miscibility in Polypropylene/Polyamide-11 Blends Journal of Molecular Structure 1012 2012: pp. 113-118. https://doi.org/10.1016/j.molstruc.2011.12.026

19. Su, B., Wu, Y.C., Jiang, L. The Art of Aligning OneDimensional (1D) Nanostructures Chemical Society Reviews 41 2012: pp. $7832-7856$. http://dx.doi.org/10.1039/C2CS35187K

20. Xiang, J., Lu, W., Hu, Y.J., Wu, Y., Yan, H. Ge/Si Nanowire Heterostructures as High-Performance Field-Effect Transistors Nature 441 2006: pp. 489-493. http://dx.doi.org/10.1038/nature04796

21. Qian, F., Gradecak, S., Li, Y., Wen, C.Y., Lieber, C.M. Core/Multishell Nanowire Heterostructures as Multicolor, High-Efficiency Light-Emitting Diodes Nano Letters 5 2005: pp. $2287-2291$. http://dx.doi.org/10.1021/n1051689e

22. Law, M., Greene, L.E., Johnson, J.C., Saykally, R., Yang, P.D. Nanowire Dye-Sensitized Solar Cells Nature Materials 4 2005: pp. 455-459. http://dx.doi.org/10.1038/nmat1387
23. Zhao, Y., He, X.L., Li, J.P., Jia, J., Gao, X.G. Enhanced Gas Sensing Properties of Aligned Porous $\mathrm{SnO}_{2}$ Nanofibers Chinese Physics Letters 29 2012: pp. 070701-1-070701-4. https://doi.org/10.1088/0256-307X/29/7/070701

24. Huang, Y., Duan, X.F., Cui, Y., Lauhon, L.J., Kim, K.H., Lieber, C.M. Logic Gates and Computation from Assembled Nanowire Building Blocks $\quad$ Science 294 2001: pp. $1313-1317$. http://dx.doi.org/10.1126/science.1066192

25. Friedman, R.S., McAlipine, M.C., Ricketts, D.S., Ham, D., Lieber, C.M. Nanotechnology: High-Speed Integrated Nanowire Circuits Nature 434 2005: pp. $1085-1085$. http://dx.doi.org/10.1038/4341085a

26. Liu, W.Y., Thomopoulos, S., Xia, Y.N. Electrospun Nanofibers for Regenerative Medicine Advanced Healthcare Materials 1 2012: pp. 10-25. http://dx.doi.org/10.1007/s10544-011-9583-x

27. Beachley, V., Katsanevakis, E., Zhang, N., Wen, X.J. Highly Aligned Polymer Nanofiber Structures Fabrication and Applications in Tissue Engineering Advance in Polymer Science 246 2012: pp. 171-212. http://dx.doi.org/10.1007/12_2011_141

28. Kim, M.S., Son, J., Lee, H.J., Hwang, H., Choi, C.H., Kim, G. Highly Porous 3D Nanofibrous Scaffolds Processed with an Electrospinning/Laser Process Current Applied Physics 14 2014: pp. 1-7. https://doi.org/10.1016/j.cap.2013.10.008

29. Mei, L.Y., Han, R., Fu, Y.Z., Liu, Y.Q. Solvent Selection for Polyacrylonitrile Using Molecular Dynamic Simulation and the Effect of Process Parameters of Magnetic-Field-Assisted Electrospinning on Fiber Alignment High Perform Polymers 27 2015: pp. 439-448. https://doi.org/10.1177/0954008314555244

30. Ma, G.P., Yang, D.Z., Nie, J. Preparation of Porous Ultrafine Polyacylonitrile (PAN) Fibers by Electrospinnning Polymers for Advanced Technologies 2009: pp. $147-150$ http://dx.doi.org/10.1002/pat.1180

31. Liu, Y.Q., Zhang, X.P., Xia, Y.N., Yang, H. Magnetic-FieldAssisted Electrospinning of Aligned Straight and Wavy Polymeric Nanofibers Advanced Materials 22 2010: pp. $2454-2457$. http://dx.doi.org/10.1002/adma.200903870

32. Zhao, Y, You, L.Y., Lu, Z.Y., Sun, C.C. Dissipative Particle Dynamics Study on the Multicompartment Micelles SelfAssembled from the Mixture of Diblock Copolymer Poly(Ethyl Ethylene) -Block - Poly(Ethylene Oxide) and Homopolymer Poly(Propylene Oxide) in Aqueous Solution Polymer 50 2009: pp. 5333-5340. https://doi.org/10.1016/j.polymer.2009.09.014

33. Jawalkar, S.S., Aminabhavi, T.M. Molecular Modeling Simulations and Thermodynamic Approaches to Investigate Compatibility/Incompatibility of Poly(L-Lactide) and Poly(Vinyl Alcohol) Blends Polymer 47 2006: pp. $8061-8071$. https://doi.org/10.1016/j.polymer.2006.09.030 